\title{
Exploratory behavior of rats following visual cortical and subcortical lesions
}

\author{
GLENDA C. MIDGLEY, KENNETH M. PRKACHIN, and RICHARD C. TEES \\ University of British Columbia, Vancouver, British Columbia, V6T IW5, Canada
}

\begin{abstract}
Exploratory behaviors of rats with lesions of the visual cortex or superior colliculus (SC) were examined using a traditional open field and a smaller closed field. Animals with large bilateral lesions of the SC or lesions restricted to the deep laminae of the SC exhibited excessive locomotor activity in both experimental situations. The degree of habituation of this hyperactivity and other behaviors such as investigating rearing, exploratory nose poking, and self-initiated exposure to illuminated visual stimuli in the closed field were also examined. Consideration of the complete range of exploratory responses and the effect of lesions on them lead to a proposal that the hyperactivity of rats with SC lesions is an impairment adjunct to sensory neglect and orienting behavior impairments.
\end{abstract}

The rat's visual system, like that of other mammalian species, is organized into independent neuronal systems mediating discreet visuomotor behaviors. Electrophysiological and behavioral evidence from a variety of mammals seems to support the proposition that the retinocortical system subserves the discriminative aspects of form vision, while a separate system subserves orientation and attention to visual stimuli (Schneider, 1969).

Attempts have been made to further dissociate the retinotectal system into discreet anatomical and functional subsystems (Casagrande, Harting, Hall, Diamond, \& Martin, 1972). In rats, as in other mammals, the tectum includes the superficial and deep laminae of the superior colliculus. The superficial laminae (stratum zonale, stratum griseum superficiale, and stratum opticum) are the terminal region of the retinal fibers, receive a substantial projection from the striate cortex, and are a source of fibers to the deep laminae. The deep laminae, however, do not receive a direct retinal input but only diffuse projections from other visual and nonvisual structures (Foreman, Goodale, \& Milner, 1978; Sprague, 1975).

This anatomical dissociation may also reflect a separate functional organization. Electrophysiological studies have demonstrated that, although the cells of the superficial laminae have a precise visuotopic map of the environment and are only respon-

The authors wish to thank Fred Valle for the use of the openfield apparatus and Rod Midgley for assistance with the histologies. This research was supported in part by National Research Council of Canada Grant APA-0179 to R. C. Tees and a Canada Council Doctoral and Killam Post Doctoral Fellowship to G. C. Midgley. Part of the research was submitted by the first author to the University of British Columbia in partial fulfillment of the requirements for the PhD degree. Requests for reprints should be sent to R. C. Tees, Department of Psychology, University of British Columbia, Vancouver, B.C., V6T IW5, Canada. sive to visual input, cells in the deep layers are driven by stimuli from all modalities and habituate rapidly to visual stimuli (Drager \& Hubel, 1975, 1976; Gordon, 1973; Horn \& Hill, 1966; Humphrey, 1968). Neurobehavioral investigation of the tree shrew has revealed that lesions which include both the superficial laminae and the deep laminae interfere with orienting behavior, while lesions confined to the superficial laminae do not (Casagrande et al., 1972). This evidence suggests that damage to the superficial laminae of the SC may result in visual deficits, while damage to the deep layers may result in more general attentive and motivational deficits.

Recently, Foreman et al. (1978), Marshall (1978), and Smith and Weldon (1976) reported that rats with lesions of the SC are excessively active in an open field. Locomotor behavior in the open field is generally assumed to measure exploratory behavior (Terry, 1979). The hyperactivity of rats with SC lesions thus has been viewed as reflecting an increase in exploratory behavior and an attempt to maintain an optimal level of stimulation (Smith \& Weldon, 1976). However, this explanation of their hyperactivity is difficult to reconcile with the well-established reports of sensory neglect and orienting behavior impairments. Rats with SC lesions do not often actively explore stimuli in the sense of orienting to them (Barnes, Smith, \& Latto, 1970; Kirvel, Greenfield, \& Meyer, 1974), and, even when they do orient to very salient visual stimuli, their orienting behavior habituates more quickly than it does in intact animals (Midgley \& Tees, 1981).

The following study was conducted to assess the involvement of the superficial and deep laminae of the SC, and the terminals of the tectocortical projections, in exploratory behavior. Locomotor activity and other exploratory behaviors are assessed in a traditional open field and a smaller, closed field in 
which exposure to illumination is self-regulated (Tees, Midgley, \& Bruinsma, 1980).

\section{METHOD}

\section{Subjects}

The subjects were 36 male Long-Evans rats (Rattus norvegicus) either purchased from the Canadian Breeding Farms and Laboratories Ltd. or bred in the UBC Biopsychology Vivarium. They were $250-350 \mathrm{~g}$ at time of surgery. The animals were assigned randomly to superior colliculus $(\mathrm{SC})$ lesion $(n=6)$, superficial superior colliculus (SP) lesion $(n=6)$, deep superior colliculus (DP) lesion $(n=6)$, striate cortex (ST) lesion $(n=6)$, striate and extrastriate cortex $(\mathrm{ST}+)$ lesion $(n=6)$, or sham $(\mathrm{SH})$ operation $(n=6)$ groups. All the animals had been tested previously in another study involving orienting to visual stimuli following water deprivation, but received ad-lib access to food and water and no handling for 2 weeks prior to open-field and closed-field testing. The animals were housed individually in standard $18 \times 20 \times 25 \mathrm{~cm}$ cages and were maintained on a 12-h-light/12-h-dark schedule throughout testing (Tees, 1968).

\section{Surgery}

Surgery was performed using a Kopf stereotaxic instrument. The rats were anesthetized with sodium pentobarbital supplemented with chloral hydrate when necessary. Midbrain lesions were made with a radio-frequency lesion maker (Radionics Model RFG4) using an electrode tip exposure of $1 \mathrm{~mm}$. The interaural line and upper edge of the incisor bar were in the horizontal plane. Superior colliculus (SC) lesions were placed $1.8 \mathrm{~mm}$ on each side of the midsagittal suture, $2.7 \mathrm{~mm}$ anterior to the most posterior aspect of the lambdoidal suture, and $3.7 \mathrm{~mm}$ below the cortical surface. The rf current was applied for $90 \mathrm{sec}$ at a tip temperature of $70^{\circ} \mathrm{C}$ (adapted from Goodale \& Murison, 1975). Superficial superior colliculus lesions (SP) were made as described above, but the electrode tip was lowered to only $3.5 \mathrm{~mm}$ below the cortical surface and a tip temperature of $55^{\circ}-60^{\circ} \mathrm{C}$ was employed. Deep superior colliculus (DP) lesions were made as above; however, the electrode was lowered to $5 \mathrm{~mm}$ below the cortical surface and a tip temperature of $55^{\circ}-60^{\circ} \mathrm{C}$ was employed. Three sham-operated animals (SH) were treated identically, but no current was applied.

Cortical lesions were produced by aspiration. The dorsal surface of the skull was removed from approximately $2 \mathrm{~mm}$ behind bregma to the lambdoidal suture and from approximately $1 \mathrm{~mm}$ lateral to the sagittal suture to the zygoma. Suction was used to remove the exposed cortical tissue under visual guidance (Tryggvason \& Tees, 1974). An attempt was made to vary the placement of the lesions to include area 17 only (ST) or areas 17 , $18,18 \mathrm{~A}$, and $7(\mathrm{ST}+)(\mathrm{Krieg}, 1946)$. Three sham-operated animals (SH) were treated identically, but no tissue was removed.

\section{Apparatus}

Open field. The open field consisted of a 1.2-m-square floor board, with .3-m-high side walls. The field was painted white, and the floor of the field was divided into 16 equal squares by black lines. The only illumination in the test room was provided by a $15-\mathrm{W}$ overhead lamp suspended $1.2 \mathrm{~m}$ above the center of the field, which maintained an illumination level of $.29 \mathrm{~cd} / \mathrm{m}^{2}$ at the center of the floor of the field.

Closed field, stimulus seeking. Three BRS/LVE translucent peeking panels were positioned in the center of three of four $16-\mathrm{cm}$ high walls surrounding a $30 \times 30 \mathrm{~cm}$ floor. The panels were positioned $1 \mathrm{~cm}$ above the floor. Visual stimuli were back-projected onto the panels by Grason-Stadler microprojectors. The visual stimuli were an outline triangle, an outline circle, and a diffuse flashing light $(10 / \mathrm{sec})$. Luminance values of the stimuli ranged between 1.2 and $2.4 \mathrm{~cd} / \mathrm{m}^{2}$ measured at the panels by a modified Weston light meter. A treadle $(3 \times 6.5 \mathrm{~cm})$ was centered on the floor immediately below each panel. The floor of the apparatus extended $6 \mathrm{~cm}$ beyond the sides of the box so that the ends of the treadle were not located within the box. The treadles were flush with the floor and separated from it by a $.3-\mathrm{cm}$ gap. A fourth treadle was centered on the floor of the fourth wall. When depressed, the treadles activated a microswitch which controlled the presentation of the visual stimuli on the corresponding panels. No visual stimulus was presented when the treadle below the fourth wall was depressed. The number and duration of treadle depressions (i.e., presses) and the presses to the panels themselves (i.e., nose pokes) were recorded, and the data collection as well as presentation of the visual stimuli were performed by solid state logic (BRS/LVE). The closed field was housed in a $66 \times 74 \times 54 \mathrm{~cm}$ sound- and light-attenuating shell.

\section{Procedure}

Open field. The animals were tested 3-4 weeks postoperatively in the open field. The animals were dark adapted for at least $4 \mathrm{~h}$ prior to testing and were tested during the dark phase of the colony's light-dark schedule (between 2100 and $2400 \mathrm{~h}$ ). The animals were moved from the colony to the testing room in light-tight carrying cases and were placed in the center of the open field, facing the corner opposite to where an experimental assistant was, who was unaware of the lesion group to which the animal belonged. The numbers of squares entered (with both front paws) and the number of rearing responses made (both front paws off the floor) were counted for a 10-min period, scored in two 5-min blocks. The number of fecal boli deposited was also recorded. The floor of the field was cleaned after each animal's test session.

Closed field, stimulus seeking. All of the animals were tested on the day following open-field testing after at least $4 \mathrm{~h}$ dark adaptation; they were tested between 2100 and $2400 \mathrm{~h}$ during the dark phase of the colony light-dark schedule. They were carried to the testing room in a light-tight carrying case and placed in the apparatus in the darkened test room.

The number and duration of treadle presses to the four individual treadles and the number of nose-poke responses to the three individual stimulus panels were recorded for a $30-\mathrm{min}$ period, in two 15-min blocks.

Histology. The animals were perfused with $.9 \%$ saline solution followed by $10 \%$ Formalin-saline while deeply anesthetized. The brains with SC lesions were blocked and freeze sectioned at either 30 or $50 \mu \mathrm{m}$. Every fifth section was saved and stained with thionin. Brains with cortical lesions were embedded in gelatin and sectioned at $30 \mu \mathrm{m}$ or were not embedded in gelatin and sectioned at $50 \mu \mathrm{m}$. Every fifth section through the lesion and every second section of the thalamus were saved and stained with cresyl violet and luxol blue.

\section{RESULTS}

\section{Behavioral Findings}

Open field. The amount of activity, rearing, and boli counts for the groups of animals were compared by analyses of variance. Differences between the groups of animals with lesions and $\mathrm{SH}$ control animals on these measures were further assessed by Dunnett's test for multiple comparisons with a control group, or by t tests when appropriate (Kirk, 1968).

The numbers of squares crossed by the groups of animals were reliably different $[F(5,30)=8.65, p<$ $.01]$. As can clearly be seen in Table 1, the SC and DP animals were more active and crossed more squares than the SH control animals $(\mathrm{p}<.01)$, while the SP animals did not differ from the SH control animals. Thus, the excessive activity recorded for SC animals did not depend upon the inclusion of the superficial layers of the colliculus in the lesions The ST and 
Table 1

Mean Number of Squares Crossed and Rearing Response Made in the Open Field by Control Animals and Animals With Lesions

\begin{tabular}{|c|c|c|c|c|c|c|}
\hline \multirow{2}{*}{$\begin{array}{l}\text { Lesion } \\
\text { Group }\end{array}$} & \multicolumn{2}{|c|}{ Block 1} & \multicolumn{2}{|c|}{ Block 2} & \multicolumn{2}{|c|}{ Total } \\
\hline & Squares & Rears & Squares & Rears & Squares & Rears \\
\hline SH & 57.17 & 30.16 & 33.33 & 16.16 & 80.5 & 46.3 \\
\hline SC & 132.33 & 9.50 & $82.33 \dagger$ & 7.83 & $214.7^{*}$ & $17.3^{*}$ \\
\hline SP & 56.33 & 10.50 & 39.33 & 7.83 & 95.7 & $18.3^{*}$ \\
\hline DP & 102.33 & 11.50 & $65.00 \dagger$ & 6.16 & $164.3^{*}$ & $17.7^{*}$ \\
\hline ST & 65.83 & 31.66 & 52.50 & 14.33 & $118.3^{*}$ & 45.9 \\
\hline ST+ & 74.33 & 31.00 & $48.16 \dagger$ & 17.50 & $122.5^{*}$ & 48.9 \\
\hline
\end{tabular}

${ }^{*} p<.01$, Dunnett's comparison with control.

$\dagger p<.01, t$ comparison between the first and second halves.

$\mathrm{ST}+$ animals also reliably crossed more squares than the SH control animals $(\mathrm{p}<.01)$.

The pattern of activity change in the two 5-min blocks varied, depending upon the lesion sustained by the animals $[F(5,30)=3.85, p<.01]$. The differences in activity between the two 5-min blocks were further assessed by $t$ tests. The SC, DP, and ST + animals crossed fewer squares in the second half of the session than in the first half $(\mathrm{p}<.01)$. The animals with SH, SP, and ST lesions also crossed fewer squares in the second half; however, the small differences between the two blocks were not statistically different for these animals. The statistically significant decrement between the two blocks for SC, DP, and ST + animals suggested that they habituated more rapidly to the open field than did SH, SP, and ST animals (see Table 1).

Reliable between-group and between-block differences were also found in the number of rearing responses $[F(5,30)=5.26, p<.01$, and $F(1,30)=31.61$, $\mathrm{p}<.01]$. The animals that sustained lesions of the superior colliculus (SC, SP, and DP) made fewer rearing responses than $\mathrm{SH}$ control animals in the open field $(\mathrm{p}<.01)$. The cortical lesion groups did not differ from SH control animals in this measure (see Table 1).

The SH, SP, ST, and ST + animals tended to have higher scores in terms of the number of boli deposited in the open field than did SC and DP animals. However, the small differences on this measure only approached statistical reliability $[\mathrm{F}(5,30)=2.17, \mathrm{p}=.08]$.

Closed field. Separate two-factor analyses of variance and multiple comparison tests were applied to the number of treadle presses, total duration of treadle presses, and nose-poke scores. The groups differed reliably on the numbers of treadle presses initiated $[F(5,30)=5.89, p<.01]$. As can be seen in Table 2, the results for these measures were very similar to the pattern of findings in the open field. The SC and DP animals were excessively active relative to $\mathrm{SH}$ control animals $(\mathrm{p}<.01)$, while the SP animals did not differ from SH control animals $(p>.05)$. The ST + animals were more active than the $\mathrm{SH}$ control animals $(\mathrm{p}<.05)$ in this apparatus; the ST animals were not $(\mathrm{p}>.05)$.

The number of treadle presses made in Blocks 1 and 2 differed for the animals with SC and DP lesions and the $\mathrm{SH}$ control animals $[\mathrm{F}(5,30)=4.39$, $\mathrm{p}<.01]$ and was also similar to the pattern of results obtained in the open field. The numbers of treadle presses by the SC, DP, and ST + animals were much higher in Block 1 than in Block $2(p<.01)$. While the numbers of treadle presses by the $\mathrm{SH}$ control and the SP and ST animals did differ reliably $(p<.01)$ between the two blocks, the differences were not as great (see Table 2).

The distribution of the total duration of presses made by the animals with lesions and the $\mathrm{SH}$ control animals differed markedly $[F(5,30)=4.75, p<.01]$. The distributions of the total time spent on each of the four treadles are presented in Table 3. Dunn's comparisons were used to assess differences in the time spent on each treadle. The SP and SH control animals spent more time on the treadle that initiated the diffuse flashing light than on any other treadle $(p<.05)$. The animals with SC, DP, ST, and ST + lesions spent more time on the treadle that did not initiate a light $(p<.01)$. They also reliably spent more time in the presence of the diffuse flashing light panel than in the presence of the circle or triangle stimulus lights $(p<.05)$.

Table 2

Mean Number of Treadle Presses Made in the Closed Field by Control Animals and Animals With Lesions

\begin{tabular}{cccl}
\hline $\begin{array}{l}\text { Lesion } \\
\text { Group }\end{array}$ & Block 1 & Block 2 & Total \\
\hline SH & 104.1 & $75.9 \dagger$ & 180.0 \\
SC & 203.8 & $97.4 \dagger$ & $301.2^{*}$ \\
SP & 116.7 & $76.5 \dagger$ & 193.3 \\
DP & 208.8 & $94.7 \dagger$ & $303.5^{*}$ \\
ST & 102.6 & $70.3 \dagger$ & 172.9 \\
ST + & 183.0 & $94.5 \dagger$ & $277.5^{*}$ \\
\hline
\end{tabular}

${ }^{*} p<.01$, Dunnett's comparison with control.

$\dagger p<.01, t$ comparison between the first and second blocks.

Table 3

Mean Duration (in Seconds) of Treadle Pressing by the Control Animals and Animals With Lesions

\begin{tabular}{lcclc}
\hline $\begin{array}{c}\text { Lesion } \\
\text { Group }\end{array}$ & $\begin{array}{c}\text { Illuminated } \\
\text { Triangle }\end{array}$ & $\begin{array}{c}\text { Illuminated } \\
\text { Circle }\end{array}$ & $\begin{array}{c}\text { Flashing } \\
\text { Light }\end{array}$ & $\begin{array}{c}\text { No } \\
\text { Light }\end{array}$ \\
\hline SH & 104.3 & 104.9 & $284.3^{*}$ & 144.9 \\
SC & 205.9 & 219.6 & 325.0 & $437.9 \dagger$ \\
SP & 105.3 & 107.7 & $305.5^{*}$ & 198.1 \\
DP & 223.9 & 213.4 & 295.2 & $419.4 \dagger$ \\
ST & 136.0 & 130.5 & 154.7 & $349.0 \dagger$ \\
ST+ & 134.3 & 128.7 & 164.6 & $380.2 \dagger$ \\
\hline
\end{tabular}

${ }^{*} p<.05$, Dunn's comparisons with the no-light treadle $\dagger p<.05$, Dunn's comparisons of no-light treadle with other treadle. 
Table 4

Mean Number of Nose Pokes to Each of the Panels by Control Animals and Animals With Lesions

\begin{tabular}{lrrrrrrr}
\hline & \multicolumn{3}{c}{ Block 1 } & & \multicolumn{3}{c}{ Block 2 } \\
\cline { 2 - 4 } \cline { 6 - 7 } & IT & IC & FL & & IT & IC & FL \\
\hline SH & 7.5 & 8.5 & 12.2 & .5 & 2.0 & .5 \\
SC & 2.7 & 2.3 & .0 & .0 & .5 & .0 \\
SP & .7 & 3.0 & .0 & .3 & .3 & .0 \\
DP & .3 & .3 & .0 & .2 & .3 & .0 \\
ST & .0 & .5 & .5 & .2 & 3.3 & .2 \\
ST+ & 1.2 & 1.3 & .5 & .3 & .5 & .3 \\
\hline
\end{tabular}

Note-IT=illuminated triangle $; I C^{\prime}=$ illuminated circle $; \quad F L=$ flashing light.

The distribution of nose pokes to the three different light-emitting panels by the animals with lesions and $\mathrm{SH}$ control animals was reliably different in Block $1[\mathrm{~F}(4,30)=2.89, \mathrm{p}<.05]$. Dunn's comparisons revealed that the $\mathrm{SH}$ control animals nosepoked each of the lighted panels reliably more often than did any of the animals with lesions $(p<.05)$. The SH control animals nose-poked the diffuse flashing light panel more than the other light-emitting panels $(\mathrm{p}<.05)$. The SH animals were not different from the animals with lesions in the second blocks. The few nose pokes by any of the animals with lesions were distributed equally among the three panels, and the small differences were not statistically reliable. These results are presented in Table 4 .

\section{Histological Findings}

Reconstructions of the subcortical lesions and drawings of the cortical lesions are presented in Figure 1. Subcortical lesions were quite uniform with respect to both size and location, and the reconstructions shown in Figure 1 are representative of the SC-, SP-, and DP-lesion groups. The cortical lesions were, however, more varied with respect to size and placement. A positive correlation between the size of the cortical lesion and excessive activity was noted for these animals. Animals with lesions confined to the striate cortex were no more active than the $\mathrm{SH}$ control animals, as indicated by average scores. A few (three cases) animals in the ST-lesion group sustained lesions that included nonstriatal tissue.

\section{DISCUSSION}

The results of this investigation confirm previous reports that damage to the SC produces excessive activity in an open field (Smith \& Weldon, 1976) and impairs directed investigatory head and rearing responses (Foreman et al., 1978; Marshall, 1978). In our study, changes in activity were found to depend upon the lesion site and upon the particular behavior examined. Directed investigatory or exploratory responses (nose pokes) were eliminated by lesions of the visual areas (visual cortex or superior colliculus). Rearing responses were eliminated by lesions of the superior colliculus (SC, SP, and DP). The superficial laminae of the SC were not implicated in changes of preferences for lights in the closed field or in excessive open- or closed-field locomotor activity. Excessive open- and closed-field activity was produced by very small lesions confined to only the ventral edge of the deep SC laminae and dorsal tegmentum and was also observed following visual cortical lesions if the lesion included a substantial portion of the tectocortical projections (extrastriate cortex and area 7, from Krieg, 1946).

The fact that rats with superficial SC lesions exhibited impaired rearing behavior but were not excessively active in the open or closed field suggests that excessive activity was not the result of sensory neglect or orienting impairments per se. The excessive activity of rats with SC lesions does not likely represent an increase in exploratory behavior or an attempt to seek stimulation and maintain an optimal level of arousal (Smith \& Weldon, 1976). Locomotor activity (number of squares crossed in the open field and number of treadles pressed in the closed field) increased, but these animals did not rear and actively explore the open field or nose-poke the stimulus

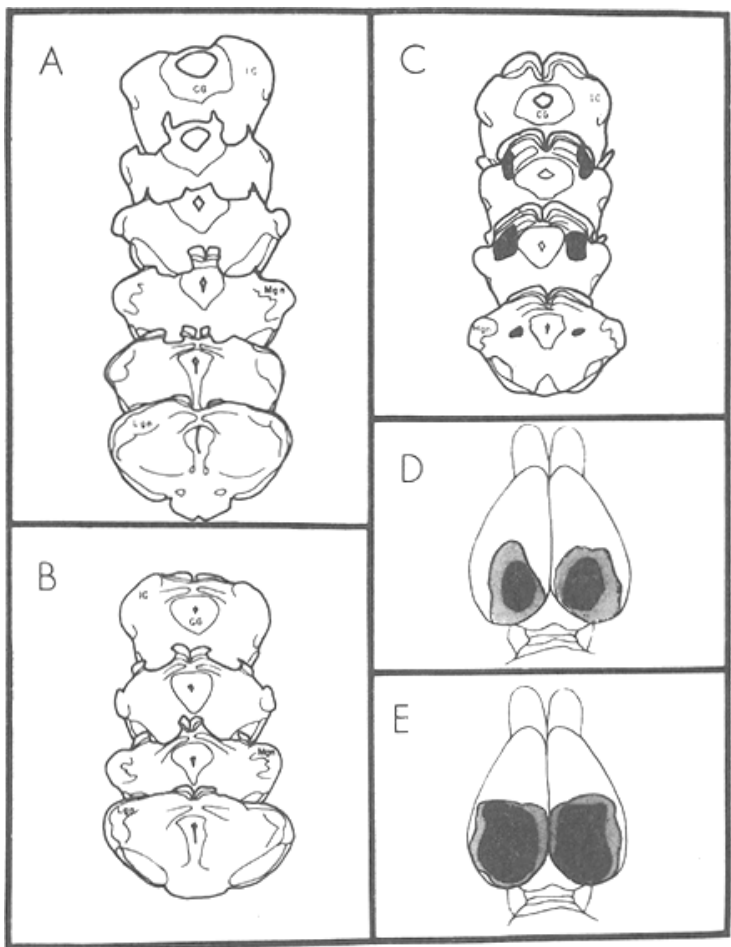

Figure 1. Histological reconstructions of a typical (A) SC lesion, (B) SP lesion, (C) DP lesion, and schematic representations of the cortical aspirations for (D) the largest and smallest ST lesion and (E) the largest and smallest ST + lesion. 
panels in the closed field. The animals with SC lesions may have increased their running in an attempt to maximize stimulus change and maintain an optimal level of arousal. However, the excessive activity of the rats with SC lesions habituated faster than SH cortical animals, and these animals did not show a preference for the flashing light-contingent treadle demonstrated by the SH control animals. In fact, they preferred the no-light contingent treadle. If the animals with SC lesions were attempting to maximize stimulus change and maintain an optimal level of arousal, one would expect a preference for the flashing light to be maintained, if not enhanced, by the lesion, not a preference for the no-light treadle. Moreover, stimulus-seeking would be expected not to habituate but to be maintained throughout the test session.

In summary, the results of this study suggest that the excessive activity of animals with SC lesions is a symptom adjunct to the well-established sensory neglect and not directly attributable to it. Rats with superficial SC lesions demonstrate sensory neglect, inasmuch as they do not actively explore stimuli by rearing and sniffing them, but they are not hyperactive. When rats with SC lesions are placed in a situation in which their hyperactivity results in increased stimulus change (the self-illuminated closed field), they do not show a preference for activity that results in a lot of stimulus change (the pattern of flashinglight-contingent treadle) but prefer activity that does not result in stimulus change (the no-light-contingent treadle). Their hyperactivity does not appear to be the result of enhanced exploration or stimulus seeking. These findings of increased activity that is adjunct to changes in exploratory behavior are compatible with previous findings of sensory neglect.

\section{REFERENCES}

Barnes, P. J., Smith, L. M., \& Latto, R. M. Orientation to visual stimuli and the superior colliculus in the rat. Quarterly Journal of Experimental Psychology, 1970, 22, 239-247.

Casagrande, V. A., Harting, J. K., Hall, W. C., Diamond, I. T., \& Martin, G. F. Superior colliculus of the tree shrew: A structural and functional subdivision into superficial and deep layers. Science, 1972, 177, 444-447.

Drager, U. C., \& Hubel, D. H. Response to visual stimulation and relationship between visual, auditory, and somesthetic inputs in mouse superior colliculus. Journal of Neurophysiology, 1975, 38, 690-713.

Drager, U., \& Hubel, D. Topography of visual and somato- sensory projections to mouse superior colliculus. Journal of Neurophysiology, 1976, 39, 91-101.

Foreman, N. P., Goodale, M. A., \& Milner, A. D. Nature of postoperative hyperactivity following lesions of the superior colliculus in the rat. Physiology \& Behavior, 1978, 21, 157-160.

Goodale, M. A., Foreman, N., \& Milner, A. D. Visual orientation in the rat: A dissociation of deficits following cortical and collicular lesions. Experimental Brain Research, 1978, 31, 445-457.

Goodale, M. A., \& Murison, R. C. C. C. The effects of lesions of the superior colliculus on locomotor orientation and the orienting reflex in the rat. Brain Research, 1975, 88, 243-255.

Gordon, B. Receptive fields in deep layers of cat superior colliculus. Journal of Neurophysiology, 1973, 36, 157-178.

HoRN, G., \& Hill, R. M. Responsiveness to sensory stimulation of units in the superior colliculus and subjacent tectotegmental regions of the rabbit. Experimental Neurology, 1966, 14, 119-223.

Humphrey, N. K. Responses to visual stimuli of units in the superior colliculus of rats and monkeys. Experimental Neurology, 1968, 20, 312-340.

KIRK, R. E. Experimental design: Procedures for the behavioral sciences. Belmont, Calif: Brooks/Cole, 1968.

Kirvel, T. D., Greenfield, R. A., \& Meyer, D. R. Multimodal sensory neglect in rats with radical unilateral posterior isocortical and superior colliculus ablations. Journal of Comparative and Physiological Psychology, 1974, 87, 156-162.

KRIEG, W. J. S. Connections of the cerebral cortex. I. The albino rat. A. Topography of the cortical areas. Journal of Comparative Neurology, 1946, 84, 221-276.

Marshall, J. F. Comparison of the sensorimotor dysfunctions produced by damage to lateral hypothalamus or superior colliculus in the rat. Experimental Neurology, 1978, 58, 203-217.

Midgley, G. C., \& Tees, R. C. Orienting behavior by rats with visual cortical and subcortical lesions. Experimental Brain Research, 1981, 41, 316-328.

Schne ider, G. E. Two visual systems. Science, 1969, 163, 895-902.

Smith, C. J., \& Weldon, D. A. Hyperactivity and deficits in problem solving following superior colliculus lesions in the rat. Physiology \& Behavior, 1976, 16, 381-385.

Sprague, J. M. Mammalian tectum: Intrinsic organization, afferent inputs, and integrative mechanisms. Neurosciences $R e$ search Program Bulletin, 1975, 13, 204-213.

TEES, R. C. Effects of early restriction on later form discrimination in the rat. Canadian Journal of Psychology, 1968, 22, 294-298.

Tees, R. C., Midgley, G., \& Bruinsma, Y. The effect of controlled rearing on the development of stimulus seeking behavior in rats. Journal of Comparative and Physiological Psychology, 1980, 94, 1003-1018.

TERRY, W. S. Habituation and dishabituation of rats' exploration of a novel environment. Animal Learning \& Behavior, 1979, 7, 525-536.

Tryggvason, S., \& Tees, R. C. Retention of three brightness discriminations by rats following posterior cortical lesions. Journal of Comparative and Physiological Psychology, 1974, 86, 637-647.

(Received for publication November 9, 1979; revision accepted January 21,1981 .) 